\title{
Varying etiologies lead to different molecular changes in Australian and South African hepatocellular carcinomas
}

\author{
NIRMITHA I. HERATH ${ }^{1}$, DAVID M. PURDIE ${ }^{2}$, MICHAEL C. KEW ${ }^{3}$, JEFFERY L. SMITH ${ }^{4}$, \\ JOANNE YOUNG ${ }^{5}$, BARBARA A. LEGGETT ${ }^{5}$ and GRAEME A. MACDONALD ${ }^{2,6}$
}

\begin{abstract}
${ }^{1}$ Leukemia Foundation of Queensland and ${ }^{2}$ Division of Population and Clinical Sciences, The Queensland Institute of Medical Research, 300 Herston Road, Brisbane 4029, QLD, Australia; ${ }^{3}$ MRC Molecular Hepatology Research Unit, University of Witwatersrand, Johannesburg, South Africa; ${ }^{4}$ Department of Biochemistry and Molecular Biology, The University of Queensland, Brisbane 4072 ; ${ }^{5}$ Conjoint Gastroenterology Laboratory, Clinical Research Centre, Royal Brisbane Hospital Research Foundation, Herston Road; ${ }^{6}$ Department of Medicine,
\end{abstract} The University of Queensland, Brisbane 4029, QLD, Australia

Received May 5, 2009; Accepted July 2, 2009

DOI: 10.3892/ijo_00000423

\begin{abstract}
There is significant regional variation in the etiologic agents responsible for hepatocellular carcinoma (HCC), which influences the genetic and epigenetic mechanisms of malignant transformation. The aim of the present study was to investigate the prevalence of allelic imbalance (AI) and $\mathrm{CpG}$ island methylation in HCCs from Australia and South Africa. Genomic DNA was extracted from malignant and non-malignant liver from 37 Australian and 24 South African HCCs and histologically normal liver from 20 transplant donors. AI was examined at 1p, 4p, 4q, 8p, 9p, 13q, 16q and $17 \mathrm{p}$, using 23 microsatellite markers. Methylation status of $p 14, p 16, p 15, R I Z 1, E$-cadherin and $O^{6}-M G M T$ was examined using methylation specific PCR, while MINTs 1, 2, 12, 25 and 31 were assessed using combined bisulfite restriction analysis. The highest prevalence of AI was observed at $9 \mathrm{p}(69 \%)$ and $17 \mathrm{p}(52 \%)$. AI was significantly higher in South African HCCs $(\mathrm{p}<0.05)$. The prevalence of promoter methylation of the six genes was significantly higher in Australian cases in both malignant and non-malignant liver tissue $(\mathrm{p}<0.05)$. MINT assays revealed an increasing degree of $\mathrm{CpG}$ island methylation in the progression of hepatocarcinogenesis which was significant for MINTs 1, 12 and 31 $(\mathrm{p}<0.05)$. MINT methylation was more prominent in Australian HCCs. These data indicate that methylation is an early event preceding malignant transformation. Methylation was more and AI less prevalent in Australian than South African
\end{abstract}

Correspondence to: Dr Nirmitha Herath, Leukemia Foundation of Queensland, The Queensland Institute of Medical Research, 300 Herston Road, Brisbane 4029, QLD, Australia

E-mail: nirmitha.herath@qimr.edu.au

Key words: allelic imbalance, methylation, hepatitis B, aflatoxin exposure, hepatocarcinogenesis
HCCs. These data suggest that there are different mechanisms of malignant transformation in HCCs from Australia and South Africa.

\section{Introduction}

Hepatocellular carcinoma (HCC) is one of the most common malignancies worldwide with an estimated annual incidence of one million cases $(1,2)$. Various genetic and epigenetic abnormalities have been identified in HCC, however hepatocarcinogenesis is poorly understood at the genomic level. A number of studies have identified chromosomal arms affected by allelic imbalance (AI) in HCCs including 1p, 4q, 5q, 6q, $8 p, 10 q, 11 p, 16 p, 16 q, 17 p$ and 22q (3-8). Two large-scale studies using multiple markers have confirmed a relatively high prevalence of $\mathrm{AI}$ in these regions $(6,7)$.

Gene silencing by $\mathrm{CpG}$ island methylation has received increasing attention as an alternate mechanism for the inactivation of tumour suppressor genes (TSGs) in HCCs. Toyota et al identified $33 \mathrm{CpG}$ islands that were methylated in colorectal cancer cell lines which were described as methylated in tumour clones (MINTS) (9). Some of these clones were methylated in both malignant and adjacent non-malignant colorectal tissue in an age-specific fashion described as type A clones, whilst another group was only methylated in the cancers (type C clones). Type C MINT clones were further sub-divided into two categories; tumours with simultaneous methylation at 3 or more loci (described as $\underline{\mathrm{C}} \mathrm{pG}$ island methylator phenotype or CIMP) and a group with a low level of methylation (9).

Currently the role of promoter methylation in hepatocarcinogenesis is not clear. Aberrant methylation of $p 16, p 15$, E-cadherin, 14-3-3- $\sigma$ and RIZ1 has been reported in HCCs and HCC cell lines (8,10-13). A significant correlation between methylation and reduced protein expression has been recognized for $p 16$, E-cadherin and $14-3-3-\sigma(8,10,11)$. While these studies provide evidence for the role of $\mathrm{CpG}$ island methylation in HCCs, these genes were analyzed in isolation or in small groups. We have recently highlighted 
fundamental differences in the prevalence of $\mathrm{CpG}$ island methylation and AI in HCCs from Australia and South Africa, with a higher prevalence of AI in South African cases, and a higher prevalence of DNA methylation in Australian HCCs (14). This presumably relate to differences in the etiopathogenesis of HCCs in the two populations, particularly the role of chronic hepatitis B infection and dietary aflatoxin exposure in the South African patients. It was not clear from these studies whether these differences were present throughout the genome or reflected more localized changes on chromosome 9p, the focus of that study.

The aims of the present study were to assess AI at a number of chromosomal arms previously shown to have significant loss of chromosomal segments or to contain candidate genes considered relevant to hepatocarcinogenesis, to examine the prevalence of $\mathrm{CpG}$ island methylation in non-malignant and malignant liver tissue and to determine the relationship between promoter methylation and AI in HCCs from Australia and South Africa. For this purpose we assessed AI at 8 chromosomal arms, 1p, 4p, 4q, 8p, 9p, 13q, 16q and 17p using microsatellite analysis. The promoter methylation status of several genes previously shown to be methylated or likely candidates for methylation in $H C C, p 14, p 16, p 15$, E-cadherin, $R I Z$ and the DNA repair gene, $\mathrm{O}^{6}$-methylguanine methyltransferase $\left(O^{6}-M G M T\right)$, were studied through methylation specific PCR. In addition, CpG islands, MINT 1, MINT 2, MINT 12, MINT 25 and MINT 31 shown to be targets for methylation in other cancers were studied using combined bisulfite restriction analysis.

\section{Patients and methods}

Patients. Malignant and adjacent non-malignant liver tissue was obtained during surgery from 37 Australian (28 Caucasian and 9 Asian) and 24 black South African patients. There were 28 males and 9 females in the Australian group with a median age of 61 years (range 28-77 years). Nine Australian patients had hemochromatosis, 6 had hepatitis $C$ virus (HCV), 6 had hepatitis B virus (HBV), one had HBV and $\mathrm{HCV}, 6$ patients had a history of excess alcohol, and 1 patient had Allagille's syndrome. Eight Australian patients had no identified underlying liver disease. Evidence of cirrhosis was seen in 21 of 37 Australian HCCs. The median age of the South African patients was 35 years (range 23-67 years) and all were male. Among the South African patients, 23 had a history of HBV infection, 21 of these also with dietary aflatoxin exposure. One South African patient had chronic HCV. Cirrhosis was present in 10 South African patients.

In addition, samples of histologically normal liver tissue from 20 transplant donors whose families had consented to this tissue being used for research purposes were also studied. There were 12 males and 8 females in the donor group with a median age of 38 (range 14-71 years). This study was approved by the Human research Ethics committees of the University of Queensland and the Queensland Institute of Medical Research.

LOH analysis. DNA was extracted and PCR microsatellite analysis was performed using a panel of 23 markers (D1S243, RIZ CA repeat, RIZ polymorphism, D4S2983, D4S1538,
D4S406, D4S426, 8p, D8S261, 8ptetra, D9S162, D9S171, D9S1752, p14 ${ }^{\mathrm{ARF}}$, D9S1748, p16 ${ }^{\mathrm{INK} 4 \mathrm{a}}$, D9S1604, D9S157, D13S1315, D16S347, D16S496, p53ivs and p53VNTR) at $1 \mathrm{p}, 4 \mathrm{p}, 4 \mathrm{q}, 8 \mathrm{p}, 9 \mathrm{p}, 13 \mathrm{q}, 16 \mathrm{q}$ and $17 \mathrm{p}$ respectively (15-17). Primer sequences were obtained from the Genome Database (http://gdbwww.gdb.org/) or from previous studies as indicated in brackets. Each microsatellite marker was amplified by PCR in a final volume of $15 \mu \mathrm{l}$ incorporating $1.5 \mu \mathrm{Ci}$ of $\left[\alpha^{-3} \mathrm{P}\right]$ dATP. The amplified products were electrophoresed on $5 \%$ denaturing polyacrylamide gels and visualised by autoradiography. A microsatellite was scored as informative if two alleles were present in the DNA from non-malignant liver tissue. AI was recorded in informative cases when there was a decrease in the intensity of one allele relative to the other. A case was considered to have AI of a chromosomal arm if one or more of the microsatellites studied in that region had evidence of $\mathrm{AI}$.

Methylation specific PCR (MSP). Methylation status of p14 (18), pl6 (19) pl5 (12), E-cadherin (20), $\mathrm{O}^{6}$-methylaguanine methyltransferase $\left(O^{6}-M G M T\right)(21)$ and $R I Z 1$ (13) promoter regions was analysed using MSP, a qualitative technique (19). DNA was bisulfite-modified using the Wizard DNA clean up system (Promega, NSW, Australia) according to the manufacturer's instructions. Blood donor DNA treated with Sss 1 methyltransferase, an enzyme that specifically catalyzes the transfer of methyl groups to cytosine residues in the cytosine-guanine doublet, was used as a positive control for each methylation assay. In addition, five human colon cancer cell lines, SW480, HCT116, LISP1, LIM1215 and LOVO were also used to aid in the optimisation of primer sequences.

Combined bisulfite restriction analysis (COBRA). Methylation of MINTs 1, 2, 12, 25 and 31 was assessed using COBRA. This assay has the advantage of allowing alterations in methylation to be assessed semi-quantitatively (22). The PCR primers for COBRA exclude $\mathrm{CpG}$ dinucleotides to enable amplification of both methylated and unmethylated templates. Bisulfite modified DNA is amplified using COBRA primers followed by restriction enzyme digests for a specific methylation site within the amplified fragment. The relative intensity of digested products was quantitated using densitometry to determine the degree of $\mathrm{CpGs}$ methylation at this site. Previously published primer sequences were used for initial amplification of these MINTs (9). Additional nested primers were necessary for MINTs 2, 12 and 25 to facilitate amplification of these $\mathrm{CG}$ rich regions. All MSP and COBRA primer sequences are presented in Table I.

Statistical analysis. $\chi^{2}$ tests (where asymptotic assumptions were satisfied), Fisher's exact tests (for small groups) or Wilcoxon rank sum tests were used to compare the prevalence of AI, promoter methylation and associated risk factors where appropriate. Mann-Whitney U tests were employed to compare the levels of methylation at MINTs, known genes, AI as well as ages of patients. Spearman's rank correlation coefficient (rs) was used to quantify the degree of association between age, AI and methylation. Multivariate regression analysis was used to compare $\mathrm{AI}$ and methylation frequencies between countries after adjusting for patient characteristics 
Table I. Oligonucleotides used for MSP and COBRA analyses.

\begin{tabular}{lll}
\hline Gene promoters & \multicolumn{1}{c}{ Forward primer } & \multicolumn{1}{c}{ Reverse primer } \\
\hline p14 & & \\
Methylated & 5'-GTGTTAAAGGGCGGCGTAGC-3' & 5'-GTGTTAAAGGGCGGCGTAGC-3' \\
Unmethylated & 5'-TTTTTGGTGTTAAAGGGTGGTGTAGT-3' & 5'-CACAAAAACCCTCACTCACAACAA-3' \\
p16 & & \\
Methylated & 5'-TTATTAGAGGGTGGGGCGGATCGC-3' & 5'-CCACTAAATCGACCTCCGACCG-3' \\
Unmethylated & 5'-TTATTAGAGGGTGGGGTGGATTGT-3' & 5'-CAACCCCAAACCACAACCATAA-3' \\
p15 & & \\
Methylated & 5'-GCGTTCGTATTTTGCGGTT-3' & 5'-CGTACAATAACCGAACGACCGA-3' \\
Unmethylated & 5'-TGTGATGTGTTTGTATTTTGTGGTT-3' & 5'-CCATACAATAACCAAACAACCAA-3' \\
E-cadherin & & \\
Methylated & 5'-GGTGAATTTTTAGTTAATTAGCGGTAC-3' & 5'-CATAACTAACCGAAAACGCCG-3' \\
Unmethylated & 5'-GGTAGGTGAATTTTTAGTTAATTAGTGGTA-3' 5'-ACCCATAACTAACCAAAAACACCA-3' \\
O'-MGMT & & \\
Methylated & 5'-TTTCGACGTTCGTAGGTTTTCGC-3' & 5'-GCACTCTTCCGAAAACGAAAC-3' \\
Unmethylated & 5'-TTTGTGTTTTGATGTTTGTAGGTTTTGT-3' & 5'-AACTCCACACTCTTCCAAAAACAAAACA-3' \\
RIZ & & \\
Methylated & 5'-GTGGTGGTTATTGGGCGACGGC-3' & 5'-GCTATTTCGCCGACCCCGACG-3' \\
Unmethylated & 5'-TGGTGGTTATTGGGTGATGGT-3' & 5'-ACTATTTCACCAACCCCAAGA-3' \\
\hline
\end{tabular}

\begin{tabular}{|c|c|c|}
\hline $\begin{array}{l}\text { MINT clones } \\
\text { and restriction } \\
\text { enzymes }\end{array}$ & Forward primer & Reverse primer \\
\hline $\begin{array}{l}\text { MINT } 1 \\
(\text { Taq } I)\end{array}$ & 5'-GGGTTGGAGAGTAGGGGAGTT-3' & 5'-AAGTTATCGAGGTAATTTTAGATGG-3' \\
\hline $\begin{array}{l}\text { MINT } 2 \\
(\text { Taq } I)\end{array}$ & $\begin{array}{l}1^{\circ} \text {-5'-YGTTATGATTTTTTTGTTTAGTTAAT-3' } \\
2^{\circ} \text {-5'-TTGTTAAATGTAAATAATT-3' }\end{array}$ & $\begin{array}{l}1^{\circ}-5^{\prime} \text {-TACACCAACTACCCAACTACCTC-3' } \\
2^{\circ}-5^{\prime} \text {-GAAAGTGTTAGAAAAATGTGTTG-3' }\end{array}$ \\
\hline $\begin{array}{l}\text { MINT } 12 \\
(\text { Mae II) }\end{array}$ & $\begin{array}{l}1^{\circ} \text {-5'-YGGGTTATGTTTTATTTTTTGTGTTT-3' } \\
2^{\circ} \text {-5'-AGTTTTTAGTAAGGTTAGTGATTTAGA-3' }\end{array}$ & $\begin{array}{l}1^{\circ}-5^{\prime} \text {-CTCAAAAAAATCAAACAACCAACCAA-3 } \\
2^{\circ} \text {-5'-TAAATTGGGAGTTTATTTAGGT-3' }\end{array}$ \\
\hline $\begin{array}{l}\text { MINT } 25 \\
(\text { Rsa I) }\end{array}$ & $\begin{array}{l}1^{\circ}-5^{\prime} \text {-GTGTTTGTAAAGGGTTGGAATTAT-3' } \\
2^{\circ} \text {-5'-GTGTTTGTAAAGGGTTGGAATTAT-3' }\end{array}$ & $\begin{array}{l}1^{\circ}-5^{\prime} \text {-AGGGTTGGATG-3' } \\
2^{\circ} \text {-5'-AGTTAGTTTTTAGTTCGGGGTTTGGT-3' }\end{array}$ \\
\hline $\begin{array}{l}\text { MINT } 31 \\
(\text { Bst } U 1)\end{array}$ & 5'-GAYGGYGTAGTAGTTATTTTGTT-3' & 5'-САТСАССАССССТСАСТТТАС-3' \\
\hline
\end{tabular}

which differed between the countries such as age, gender, and risk factors such as $\mathrm{HBV}, \mathrm{HCV}$ and hemochromatosis. All statistical analyses were performed using SPSS for Windows release 11.0 , and a $\mathrm{p}<0.05$ was considered statistically significant.

\section{Results}

We examined DNA isolated from paired non-malignant and malignant liver tissue from 61 patients for evidence of AI, using a panel of 23 polymorphic microsatellite markers. All 61 HCCs analysed were informative for at least one marker. The highest prevalence of AI were observed at chromosomal arms, 1p (41\%), 4q (49\%), 8p (41\%), 9p (69\%) and 17p (52\%). The prevalence of AI was consistently higher in the South African HCCs at each chromosomal region tested (Fig. 1), with the median LOH being significantly higher in South African HCCs compared to Australian HCCs $(p<0.0034$, Wilcoxon rank sum test). When specific regions were examined, the rate of $\mathrm{AI}$ at $9 \mathrm{p}(83 \%$ vs $59 \%)$ and $16 \mathrm{q}(50 \%$ 


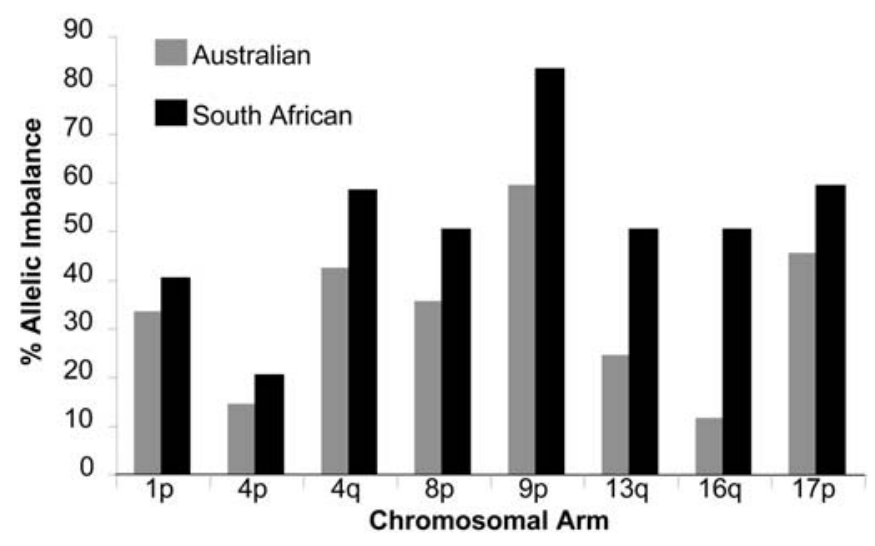

Figure 1. Prevalence of AI in Australian and South African HCCs at each chromosomal arm examined. ${ }^{*} \mathrm{p}<0.05$.

vs $11 \%$ ) were significantly higher in the South African HCCs compared to the Australian HCCs $\left(\mathrm{p}<0.05, \chi^{2}\right.$ ) (Figs. 2 and 3). A summary of the LOH data is provided in Table II. Interestingly, patients with HBV were more likely to have AI affecting more than 3 chromosomal regions $\left(p<0.0001, \chi^{2}\right)$. $\mathrm{LOH}$ was not associated with cirrhosis, hepatitis $\mathrm{C}$ virus infection or hemochromatosis.
The methylation status of the promoter regions of six genes, p16, p14, p15, E-cadherin, RIZ1 and $O^{6}$-MGMT, was assessed by MSP (Fig. 4). The prevalence of methylation of these genes in normal, non-malignant and malignant liver is presented in Table II. Promoter methylation was detected at RIZ1 in $16(26 \%) \mathrm{HCCs}$, at pl6 in $18(30 \%)$, at p15 in 35 (57\%), at p14 in $24(39 \%)$ and E-cadherin in 14 (23\%) HCCs. Evidence of promoter methylation in non-malignant liver was detected at RIZ1 in 3 cases (5\%), at p16 in $4(7 \%)$, at $p 15$ in $33(54 \%)$, at p14 in $19(31 \%)$ and at E-cadherin in 23 cases $(38 \%)$. Methylation was considerably lower in histologically normal livers obtained from organ donors (Table III). No methylation was observed at $O^{6}-M G M T$.

The median MSP value of these 6 genes was significantly higher in the Australian HCCs compared to the South African HCCs $(p=0.02$, Wilcoxon rank sum test). This was also observed in non-malignant liver where promoter methylation was higher in Australian compared to South African cases ( $\mathrm{p}<0.001$, Wilcoxon rank sum test). A sub-group of HCCs had no methylation at any of the $6 \mathrm{CpG}$-island tested. This was more likely to occur in South African than Australian HCCs $\left(38 \%\right.$ vs $3 \%$ respectively, $\left.\mathrm{p}<0.01, \chi^{2}\right)$.

Promoter methylation in HCCs was more prevalent in patients with cirrhosis than in those without (39\% vs 30\%),

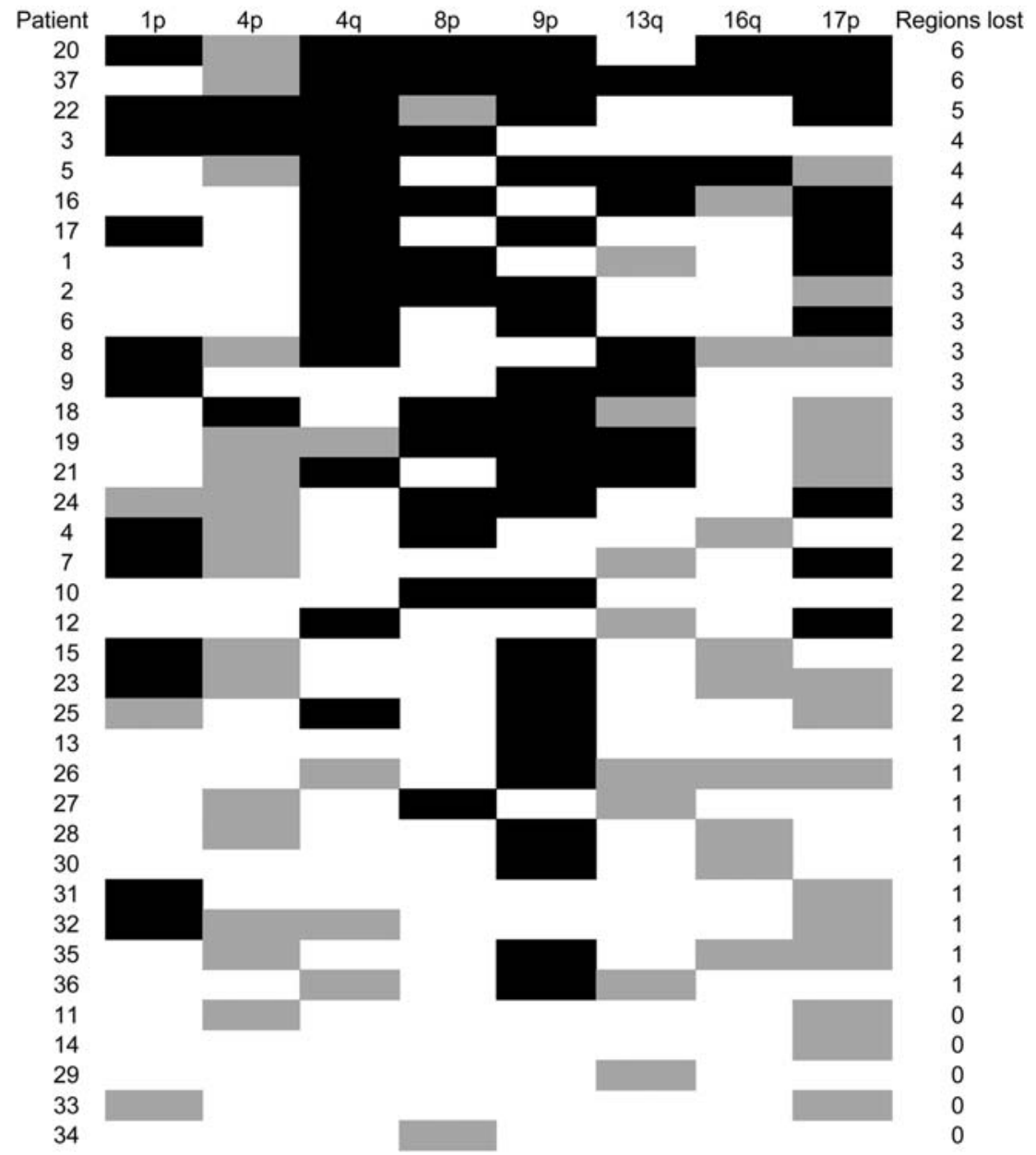

Figure 2. The frequency of chromosomal regions lost amongst Australian patients. ${ }^{*}$ The cases are recorded in descending order based on the prevalence of AI. Black lanes represent LOH of that particular chromosomal region. Grey lanes represent cases that were not informative. The numbers in the last column indicates the number of chromosomal regions lost collectively in each patient. 


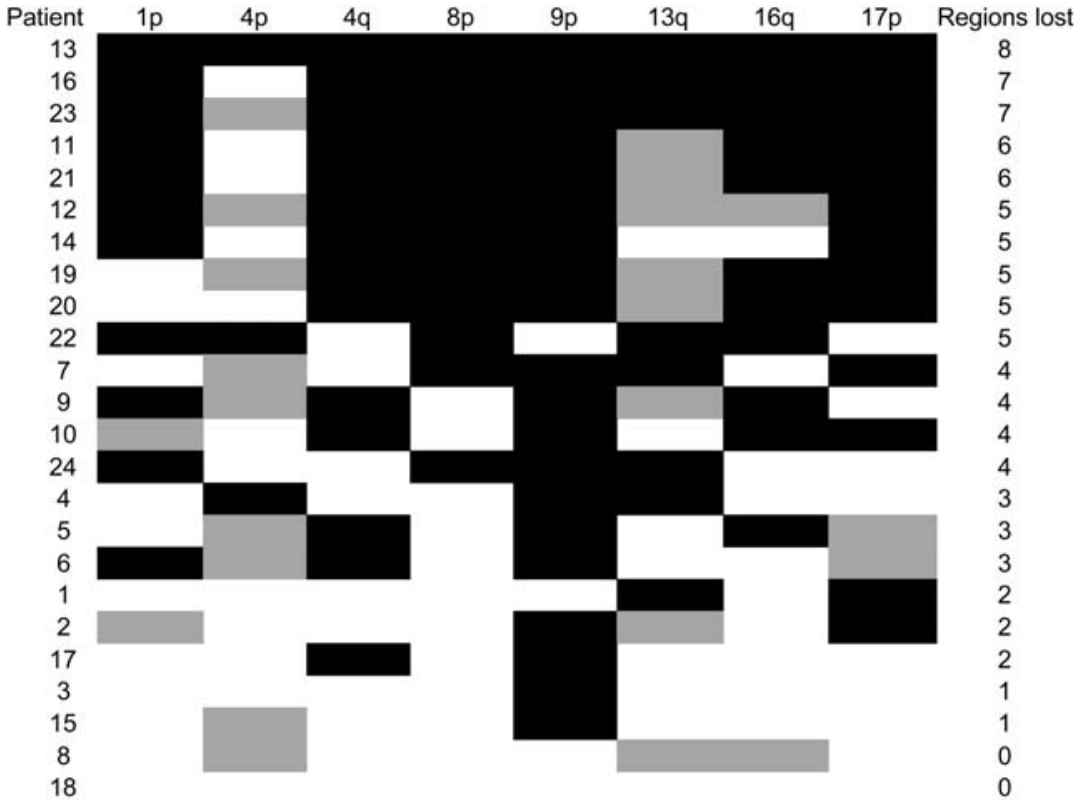

Figure 3. The frequency of chromosomal regions lost amongst South African patients. *The cases are recorded in descending order based on the prevalence of AI. Black lanes represent $\mathrm{LOH}$ of that particular chromosomal region. Grey lanes represent cases that were not informative. The numbers in the last column indicates the number of chromosomal regions lost collectively in each patient.

Table II. A summary of chromosomal instability at microsatellite markers used in chromosomal regions examined.

\begin{tabular}{|c|c|c|}
\hline $\begin{array}{l}\text { Chromosomal } \\
\text { region }\end{array}$ & Microsatellite & $\begin{array}{c}\text { Prevalence of AI } \\
(\%)\end{array}$ \\
\hline \multirow{3}{*}{$1 \mathrm{p}$} & D1S243 & $17 / 47(36)$ \\
\hline & RIZ CA repeat & $8 / 44(18)$ \\
\hline & RIZ polymorphism & $12 / 28(43)$ \\
\hline $4 p$ & D4S2983 & $6 / 36(17)$ \\
\hline \multirow[t]{3}{*}{$4 q$} & D4S1538 & $17 / 40(43)$ \\
\hline & D4S406 & $15 / 41(37)$ \\
\hline & D4S426 & $19 / 46(41)$ \\
\hline \multirow[t]{3}{*}{$8 p$} & $8 p$ & $9 / 32(28)$ \\
\hline & D8S261 & $14 / 44(32)$ \\
\hline & $8 \mathrm{p}$ tetranucleotide & $16 / 42(38)$ \\
\hline \multirow[t]{8}{*}{$9 \mathrm{p}$} & D9S162 & $15 / 42(36)$ \\
\hline & D9S171 & $9 / 36(25)$ \\
\hline & D9S1752 & $7 / 31(23)$ \\
\hline & p14 intragenic & $10 / 47(21)$ \\
\hline & D9S1748 & $8 / 42$ (19) \\
\hline & p16 intragenic & $12 / 43(28)$ \\
\hline & D9S1604 & $11 / 16(69)$ \\
\hline & D9S157 & $12 / 29(41)$ \\
\hline $13 q$ & D13S1315 & $15 / 45(33)$ \\
\hline \multirow[t]{2}{*}{$16 \mathrm{q}$} & D16S347 & $8 / 36(22)$ \\
\hline & D16S496 & $9 / 30(30)$ \\
\hline \multirow[t]{2}{*}{$17 p$} & p53ivs & $15 / 40(38)$ \\
\hline & p53VNTR & $18 / 40(45)$ \\
\hline
\end{tabular}

however this difference was not statistically significant. Furthermore, methylation of non-malignant but not malignant liver was also detected in p14 in 11 cases, p15 in 10 , $E$-cadherin in 16 and $p 16$ in two. The prevalence of promoter methylation of $p 14, p 15$ and E-cadherin was frequent in non-malignant liver tissue than p16 and RIZ1 promoter methylation.

The relationship between $\mathrm{LOH}$ and promoter methylation detected by MSP in Australian and South African HCCs is shown in Fig. 5. The HCCs appear to fall into separate groups, with greater levels of AI in South African cases and promoter methylation more prominent in the Australian cases.

When these two factors were correlated with age, there was an inverse correlation between AI and age ( $r=-0.29$, $\mathrm{p}=0.02$ ) and a positive correlation between methylation and age $(r=0.33, p=0.01)$. Once these values were re-adjusted for differences in patient characteristics between the two countries such as gender and risk factors, there was still a significant difference in the frequency of AI between Australia and South Africa $(\mathrm{p}=0.03)$. However, no significant difference was observed in the frequency of methylation between the two countries $(\mathrm{p}=0.55)$.

The average proportion of methylation in HCCs for all five MINT clones was 9\%. Methylation of both malignant and non-malignant liver was observed at MINTs 1, 12 and 25 (Table IV). The distribution of methylation was skewed with a significant proportion of cases having no methylation. MINT 1 had the highest degree of methylation, particularly in malignant liver (61\%), whilst MINT 2 had the lowest. Methylation at MINTs 2 and 31 was only detected in malignant liver tissue.

In general, there were increasing levels of MINT methylation in the progression from normal donor liver, through non-malignant to malignant liver tissue. This was statistically significant at MINT 1 (normal vs non-malignant, $\mathrm{p}<0.001$, 

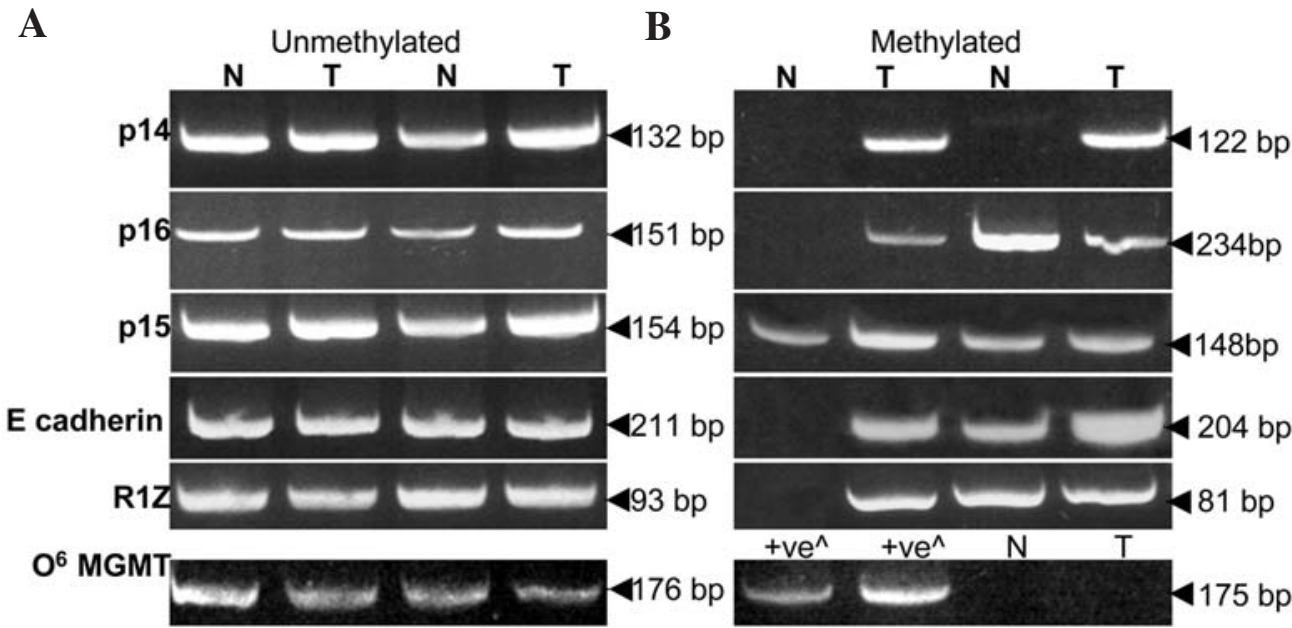

Figure 4. MSP analysis of multiple CpG islands in HCCs. (A) PCR amplified, bisulfite modified DNA using unmethylation specific primers. The presence of a band (arrow) indicates the successful bisulfite modification of both the non-malignant $(\mathrm{N})$ and the corresponding HCC (T). (B) PCR amplified, bisulfite modified DNA using methylation specific primers. The presence of a band indicates the presence of promoter methylation, whereas the absence of a band indicates a lack of promoter methylation.

Table III. The prevalence and percentage of methylation in normal, non-malignant and malignant liver at RIZ, p16, p15, p14, E-cadherin and $O^{6}-M G M T$.

\begin{tabular}{lcccccc}
\hline Gene & RIZ & $p 16$ & $p 15$ & $p 14$ & E-cadherin & $O^{6}-M G M T$ \\
\hline $\begin{array}{l}\text { Normal (n=20) } \\
\text { Cases methylated (\%) }\end{array}$ & $1(5)$ & $3(15)$ & $4(20)$ & $2(10)$ & $2(10)$ & $0(0)$ \\
$\begin{array}{c}\text { Non-malignant (n=61) } \\
\text { Cases methylated (\%) }\end{array}$ & $3(5)$ & $4(7)$ & $33(54)$ & $19(31)$ & $23(38)$ & $0(0)$ \\
$\begin{array}{l}\text { Malignant (n=61) } \\
\text { Cases methylated (\%) }\end{array}$ & $16(26)$ & $18(28)$ & $35(57)$ & $24(39)$ & $14(23)$ & $0(0)$ \\
\hline
\end{tabular}

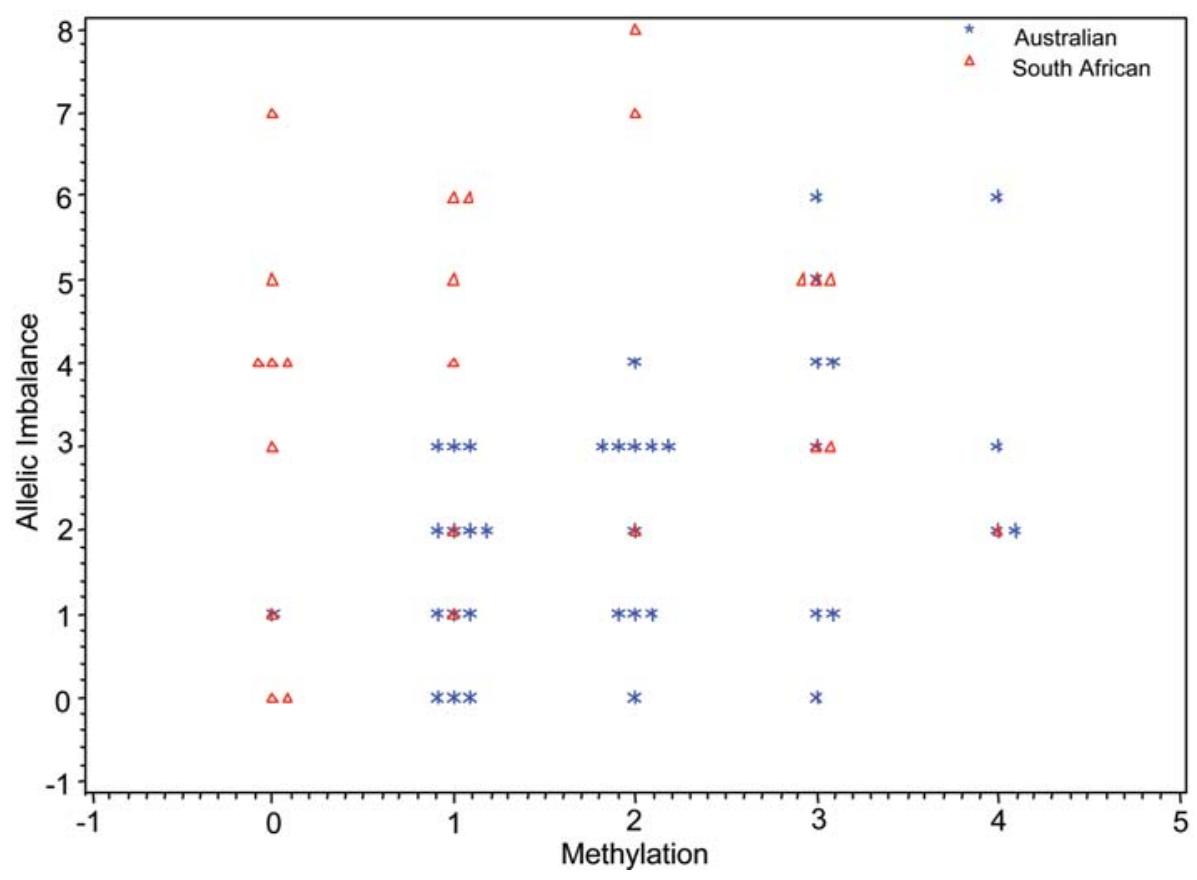

Figure 5. AI and $\mathrm{CpG}$ island methylation in Australian and South African HCCs. 
Table IV. The prevalence, mean and range of methylation observed in normal, non-malignant and malignant liver at MINTs 1, $2,12,25$ and 31 .

\begin{tabular}{|c|c|c|c|c|c|}
\hline MINT clones & MINT $1^{\mathrm{a}}$ & MINT 2 & MINT $12^{\mathrm{b}}$ & MINT 25 & MINT $31^{\mathrm{c}}$ \\
\hline \multicolumn{6}{|l|}{ Normal $(n=20)$} \\
\hline No. of cases methylated ${ }^{\mathrm{d}}$ & 0 & 0 & 0 & 3 & 0 \\
\hline Mean $(\%)$ & 0.75 & 0 & 0 & 2 & 0 \\
\hline Range (\%) & $0-3$ & $0-0$ & $0-0$ & $0-14$ & $0-0$ \\
\hline \multicolumn{6}{|l|}{ Non-malignant $(\mathrm{n}=61)$} \\
\hline No. of cases methylated ${ }^{\mathrm{d}}$ & 15 & 0 & 5 & 4 & 0 \\
\hline Mean $(\%)$ & 6.45 & 0 & 2.5 & 4.6 & 0.1 \\
\hline Range (\%) & $0-35$ & $0-0$ & $0-39$ & $0-98$ & $0-3$ \\
\hline \multicolumn{6}{|l|}{ Malignant $(n=61)$} \\
\hline No. of cases methylated ${ }^{\mathrm{d}}$ & 37 & 4 & 12 & 8 & 13 \\
\hline Mean $(\%)$ & 21 & 1.6 & 7.3 & 6 & 8 \\
\hline Range (\%) & $0-92$ & $0-55$ & $0-66$ & $0-97$ & $0-88$ \\
\hline
\end{tabular}

${ }^{a} \mathrm{~A}$ significant difference in the levels of methylation was seen between normal, non-malignant and malignant liver ( $\mathrm{p}<0.001$, Mann-Whitney $\mathrm{U}$ test). ${ }^{\mathrm{b}} \mathrm{A}$ significant difference in the levels of methylation was seen between normal, non-malignant and malignant liver ( $\mathrm{p}<0.05$, MannWhitney U test). ${ }^{\mathrm{c}} \mathrm{A}$ significant difference in the levels of methylation was seen between normal, non-malignant and malignant liver $(\mathrm{p}<0.05$, Mann-Whitney U test). ${ }^{\mathrm{d}}$ As discussed in the text, the mean methylation at all MINTs in the HCCs was $9 \%$. A case was considered to have evidence of methylation for any particular MINT if it was $>9 \%$ methylated at that MINT.

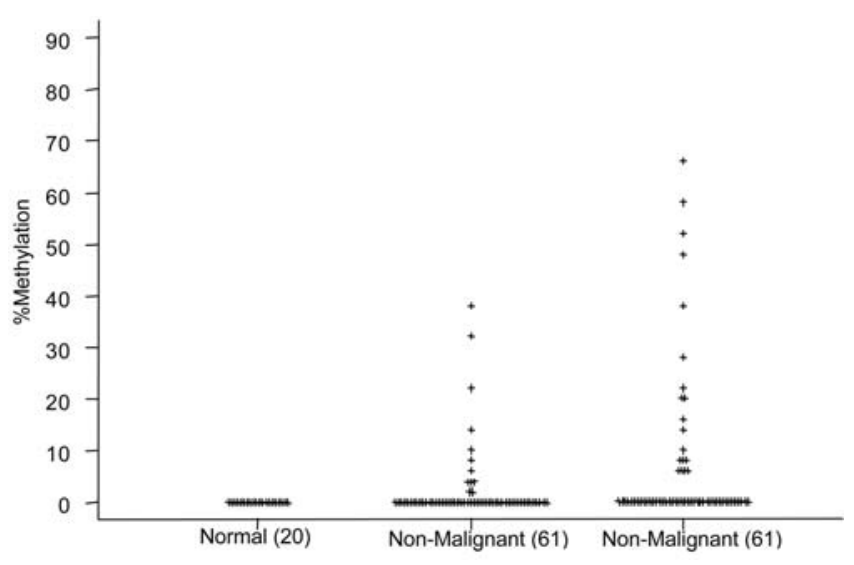

Figure 6. Progressive increase in the degree of methylation at MINT 12.

non-malignant vs malignant, $\mathrm{p}<0.001$, Mann-Whitney $\mathrm{U}$ test), MINT 12 (non-malignant vs malignant, $\mathrm{p}<0.05$, MannWhitney U test) and MINT 31 (non-malignant vs malignant, $\mathrm{p}<0.05$, Mann-Whitney U test) (Fig. 6). The level of methylation was again higher in the Australian HCCs compared to the South African HCCs, however this was not statistically significant.

\section{Discussion}

There are few integrated data in HCCs on CpG island methylation at multiple loci and the relationship between this and
AI. In the present study some of these issues have been assessed by examining a series of $61 \mathrm{HCCs}$ for AI on 8 chromosomal arms previously identified as potential targets for AI in HCC (1p, 4p, 4q, 8p, 9p, 13q, 16q, 17p). The results confirm our previous observation (14) that $\mathrm{AI}$ is more prevalent in HCCs from South Africa compared to Australian cases. In that earlier study only one chromosomal region on 9p was studied. The present study extends those observations, examining 8 chromosomal arms and at each of these the prevalence of AI was higher in the South African patients. While this may be a feature related to the chromosomal regions selected for study, the consistency of this observation suggests it is a generalized phenomenon.

The reasons for the greater degree of AI in South African HCCs, at least when compared to Australian cases, presumably relate to the pathogenesis of these cancers in the two populations. The majority of the South African patients with HCC in the present study had chronic HBV infection and were from regions with high dietary aflatoxin exposure, factors previously found to be associated with AI $(23,24)$. These risk factors for $\mathrm{HCC}$ were not jointly present in the Australian patients. Genetic factors may also have contributed to the differences in the biology of the HCCs from the two countries.

With the methodology used in the present study and the tissue available it was not possible to determine if AI was present in liver tissue from transplant donors or the nonmalignant liver tissue adjacent to the HCC. To detect AI in these tissues a source of germ line DNA (such as peripheral blood leukocytes) would be required. 
In contrast to $\mathrm{AI}$, the prevalence of $\mathrm{CpG}$ methylation (as assessed by MSP) was higher in the Australian HCCs compared to South African cases. Non-malignant liver also showed a greater prevalence of promoter methylation in Australian compared to South African cases, while methylation was virtually absent in normal donor liver tissue.

As with AI, the reason for the differences in the prevalence of methylation in HCCs from the two countries presumably relates to host and environmental factors that contribute to malignant transformation in the liver. One important consideration is the effect of patient age. Australian patients were significantly older than those from South Africa $(\mathrm{p}<0.001$, Mann-Whitney $\mathrm{U}$ test) and this may have contributed to the greater prevalence of methylation in the Australian population. All genes selected for study by MSP have previously been implicated in carcinogenesis in a variety of tissues. Epigenetic silencing of these and other TSGs by promoter methylation during the aging process may contribute to the recognised association of increased HCC risk with advancing age.

We have previously shown that p14 appears to be methylated in an age-dependent fashion in Australian HCCs (14). The prevalence of methylation of the promoters of $p 15$ and E-cadherin in non-malignant liver in the present study suggests that these $\mathrm{CpG}$ islands are also methylated in an age-dependent fashion. p16 and RIZ1 promoter methylation was principally only detected in malignant tissue consistent with the current understanding that these are subject to cancer-specific methylation (13).

An important observation from the present study was the prevalence of $\mathrm{CpG}$ island methylation in non-malignant liver tissue. The MSP studies demonstrated increasing prevalence, while data from MINT clones revealed an increasing degree of $\mathrm{CpG}$ island methylation in the progression from histologically normal liver tissue, through non-malignant liver tissue to malignant liver. These data imply that methylation is an early pre-malignant event in hepatocarcinogenesis. They also provide evidence of a field effect with potentially significant pre-malignant alterations in the non-malignant liver tissue of patients with HCC. Further study is required, but the presence of DNA methylation in liver tissue may be a useful marker of increased risk of malignant transformation in individuals with chronic liver disease.

Cirrhosis is a recognised risk factor for HCC. Somewhat surprisingly, methylation in non-malignant liver did not correlate with the presence of cirrhosis. The reasons for this were not clear. However, if the mechanisms of malignant transformation are fundamentally different in the two populations then methylation may not be a feature of cirrhosis in the South African patients. This would potentially mask any relationship between cirrhosis and methylation in the Australian cases.

Kondo et al observed frequent methylation at MINTs 1, 2, 12 and 31 in a series of micro-dissected HCCs from Japan (25). In the present study the highest levels of methylation were observed at MINT 1 and the lowest level of methylation was observed at MINT 2, while Kondo et al found the greatest methylation at MINT 2 and the lowest at MINT 25 (25). These data again emphasize the contribution of regional factors to genomic alterations and the pathogenesis of HCC.
Additionally, several cases had methylation in DNA from non-malignant liver tissue but not in the corresponding HCCs. Similar observations have been reported in other tissues including lung, colon and breast cancers (13,26-28).

This study demonstrates that HCCs in Australia occur later in life in the setting of hypermethylation. In contrast, HCCs in South Africa occur early in life as a result of AI which does not appear to play a major role in Australian HCCs. South African HCCs may demonstrate hypermethylation with age however, in view of the levels of AI observed in South African HCCs, hypermethylation of genes does not appear to be required for hepatocarcinogenesis in this group. The reasons for the differences observed between the two groups are not clear but likely relate to the etiopathogenesis of HCC in both countries.

Until recently, HCCs have been treated as a single entity. Although histologically they appear to be the same, differences in the onset of the disease and the pathobiology of the cancer with implications for differing etiologies suggest that these are in fact two different types of cancers. This has been previously demonstrated for the TSG $p 53$. Data from the present study show similar trends however, this is a broader study confirming the differences between the two types of HCCs. These data emphasize the role of host and environmental factors on the mechanisms of hepatocarcinogenesis and regulatory pathways affected in different regions of the world. These issues need to be considered in the interpretation of studies of the biology of HCC.

\section{Acknowledgements}

This study was supported by grands from the Variety Club Medical Research Scholarship and the Royal Brisbane Hospital Research Foundation Research Committee (to N. Herath).

\section{References}

1. Di Bisceglie AM, Rustgi VK, Hoofnagle JH, Dusheiko GM and Lotze M: NIH conference; hepatocellular carcinoma. Ann Intern Med 108: 390-401, 1988.

2. Parkin DM, Stjernsward J and Muir CS: Estimates of the worldwide frequency of twelve major cancers. Bull World Health Organ 62: 163-182, 1984.

3. Hammond C, Jeffers L, Carr Bi and Simon D: Multiple genetic alterations, 4q28, a new suppressor region, and potential gender differences in human hepatocellular carcinoma. Hepatology 29: 1479-1485, 1999.

4. Piao Z, Park C, Park JH and Kim H: Deletion mapping of chromosome $4 \mathrm{q}$ in hepatocellular carcinoma. Int J Cancer 79: 356-360, 1998.

5. Fujiwara Y, Ohata H, Emi M, et al: A 3-Mb physical map of the chromosome region 8 p21.3-p22, including a $600-\mathrm{kb}$ region commonly deleted in human hepatocellular carcinoma, colorectal cancer, and non-small cell lung cancer. Genes Chromosomes Cancer 10: 7-14, 1994.

6. Nagai H, Pineau P, Tiollais P, Buendia MA and Dejean A: Comprehensive allelotyping of human hepatocellular carcinoma. Oncogene 14: 2927-2933, 1997.

7. Boige V, Laurent-Puig P, Fouchet $\mathrm{P}$, et al: Concerted nonsyntenic allelic losses in hyperploid hepatocellular carcinoma as determined by a high-resolution allelotype. Cancer Res 57: 1986-1990, 1997.

8. Iwata N, Yamamoto H, Sasaki S, et al: Frequent hypermethylation of $\mathrm{CpG}$ islands and loss of expression of the 14-3-3 sigma gene in human hepatocellular carcinoma. Oncogene 19: 5298-5302, 2000 
9. Toyota M, Ahuja N, Ohe-Toyota M, et al: $\mathrm{CpG}$ island methylator phenotype in colorectal cancer. Proc Natl Acad Sci USA 96: 8681-8686, 1999.

10. Matsuda Y, Ichida T, Matsuzawa J, Sugimura K and Asakura H: p16(INK4) is inactivated by extensive CpG methylation in human hepatocellular carcinoma. Gastroenterology 116: 394-400, 1999.

11. Kanai Y, Ushijima S, Hui AM, et al: The E-cadherin gene is silenced by CpG methylation in human hepatocellular carcinomas. Int J Cancer 71: 355-359, 1997.

12. Wong IH, Lo YM, Yeo W, Lau WY and Johnson PJ: Frequent p15 promoter methylation in tumor and peripheral blood from hepatocellular carcinoma patients. Clin Cancer Res 6: 3516-3521, 2000.

13. Du Y, Carling T, Fang W, et al: Hypermethylation in human cancers of the RIZ1 tumor suppressor gene, a member of a histone/protein methyltransferase superfamily. Cancer Res 61: 8094-8099, 2001.

14. Herath NI, Kew MC, Walsh MD, et al: Reciprocal relationship between methylation status and loss of heterozygosity at the p14ARF locus in Australian and South African hepatocellular carcinomas. J Gastroenterol Hepatol 17: 301-307, 2002.

15. Biden K, Young J, Buttenshaw R, et al: Frequency of mutation and deletion of the tumor suppressor gene CDKN2A (MTS1/p16) in hepatocellular carcinoma from an Australian population. Hepatology 25: 593-597, 1997.

16. Liew CT, Li HM, Lo KW, et al: High frequency of p16 $6^{\mathrm{INK} 4 \mathrm{~A}}$ gene alterations in hepatocellular carcinoma. Oncogene 18: 789-795, 1999

17. Herranz M, Urioste M, Santos J, et al: Analysis of the INK4a/ARF locus in non-Hodgkin's lymphomas using two new internal microsatellite markers. Leukemia 13: 808-810, 1999.

18. Esteller M, Tortola S, Toyota M, et al: Hypermethylation-associated inactivation of p14(ARF) is independent of p16(INK4a) methylation and p53 mutational status. Cancer Res 60: 129-133, 2000 .

19. Herman JG, Graff JR, Myohanen S, Nelkin BD and Baylin SB: Methylation-specific PCR: a novel PCR assay for methylation status of CpG islands. Proc Natl Acad Sci USA 93: 9821-9826, 1996.
20. Tamura G, Yin J, Wang S, et al: E-Cadherin gene promoter hypermethylation in primary human gastric carcinomas. J Natl Cancer Inst 92: 569-573, 2000.

21. Esteller M, Toyota M, Sanchez-Cespedes M, et al: Inactivation of the DNA repair gene O6-methylguanine-DNA methyltransferase by promoter hypermethylation is associated with $\mathrm{G} \rightarrow \mathrm{A}$ mutations in K-ras in colorectal tumorigenesis. Cancer Res 60: 2368-2371, 2000.

22. Xiong Z and Laird PW: COBRA: a sensitive and quantitative DNA methylation assay. Nucleic Acids Res 25: 2532-2534, 1997.

23. Kew MC: Clinical, pathologic, and etiologic heterogeneity in hepatocellular carcinoma: evidence from southern Africa. Hepatology 1: 366-369, 1981.

24. Tokino T, Tamura H, Hori N and Matsubara K: Chromosome deletions associated with hepatitis B virus integration. Virology 185: 879-882, 1991.

25. Kondo Y, Kanai Y, Sakamoto M, Mizokami M, Ueda R and Hirohashi S: Genetic instability and aberrant DNA methylation in chronic hepatitis and cirrhosis-A comprehensive study of loss of heterozygosity and microsatellite instability at 39 loci and DNA hypermethylation on $8 \mathrm{CpG}$ islands in microdissected specimens from patients with hepatocellular carcinoma. Hepatology 32: 970-979, 2000.

26. Esteller M, Sanchez-Cespedes M, Rosel LR, et al: Detection of aberrant promoter hypermethylation of tumor suppressor genes in serum DNA from non-small cell lung cancer patients. Cancer Res 59: 67-70, 1999.

27. Zochbauer-Muller S, Fong KM, Virmani AK, et al: Aberrant promoter methylation of multiple genes in non-small cell lung cancers. Cancer Res 61: 249-255, 2001.

28. Belinsky SA, Nikula KJ, Palmisano WA, et al: Aberrant methylation of p16(INK4a) is an early event in lung cancer and a potential biomarker for early diagnosis. Proc Natl Acad Sci USA 95: 11891-11896, 1998. 